\title{
Direct bilirubin levels are prognostic in non-small cell lung cancer
}

\author{
Ying-Jian Song ${ }^{1}$, Xin-Huai Gao ${ }^{1}$, Yong-Qing Hong ${ }^{1}$ and Li-Xin Wang ${ }^{1}$ \\ ${ }^{1}$ Department of Respiratory Medicine, Huai'an First People's Hospital, Nanjing Medical University, Huai'an 223300, Jiangsu, \\ China \\ Correspondence to: Li-Xin Wang, email: wlxhunter923@163.com \\ Keywords: non-small cell lung cancer; bilirubin; chemotherapy; outcome; nomogram
}

Received: October 06, $2017 \quad$ Accepted: November 16, $2017 \quad$ Published: December 12, 2017

Copyright: Song et al. This is an open-access article distributed under the terms of the Creative Commons Attribution License 3.0 (CC BY 3.0), which permits unrestricted use, distribution, and reproduction in any medium, provided the original author and source are credited.

\section{ABSTRACT}

We investigated the prognostic value of serum bilirubin levels in stage I-II non-small cell lung cancer (NSCLC) patients and evaluated the relationship between bilirubin levels and response to first-line platinum-based chemotherapy. We divided 634 NSCLC patients from a single hospital in China into retrospective training $(n=307)$ and prospective validation $(n=327$ ) cohorts. X-tile was used to identify the optimal serum bilirubin cutoff value for sorting retrospective cohort patients into low and high overall survival (OS) groups. TNM stage and serum bilirubin levels were associated with OS on univariate analysis. Direct bilirubin (DBIL) levels were correlated with tumor progression and response to first-line platinum-based chemotherapy, and were associated with OS after adjusting for TNM stage. Our findings indicate a DBIL-based prognostic nomogram is more accurate than the TNM staging system in predicting clinical outcomes, and that the DBIL level is an independent predictor of OS in NSCLC. Thus, an index that combines DBIL with TNM stage may better predict patient outcomes than TNM stage alone.

\section{INTRODUCTION}

Lung cancer is the leading cause of cancer death worldwide [1]. Non-small cell lung cancer (NSCLC) accounts for approximately $85 \%$ of lung cancer cases in the U.S. [2]. Predicting NSCLC patient outcomes is challenging due to differences in genetic, clinical, and environmental factors [3]. Many NSCLC patients are diagnosed at a late stage and experience relapse and disease progression following surgical resection [4].

Prognostic predictions in NSCLC are based on the tumor-node-metastasis (TNM) staging system. However, this system cannot account for several factors that impact patient outcomes including clinical characteristics, lab results, and treatment regimens. Prediction tools such as nomograms have been developed to predict prognosis in patients with various cancers. However, they are not routinely used in NSCLC.

Serum bilirubin is an end product of heme metabolism. It has been shown to have potent antioxidant and antitumor effects in lung cancer [5, 6]. Studies of the relationships between circulating antioxidant levels and cancer risk have yielded conflicting results. Baseline serum bilirubin levels were inversely correlated with cancer mortality in a Belgian population [7]. Additionally, two prospective studies reported an inverse association between serum bilirubin levels and lung cancer risk [8, 9].

In this study, we explored the relationship between serum bilirubin levels and clinical outcomes following surgical resection in early-stage (I-II) NSCLC patients. We also evaluated the association between serum bilirubin levels and response to first-line platinum-based chemotherapy. Finally, we developed a serum bilirubinbased nomogram that could more accurately predict 5-year clinical outcomes in these patients than TNM stage alone.

\section{RESULTS}

\section{Patient characteristics}

NSCLC patients from a single hospital in China were divided into retrospective training $(n=307)$ and prospective validation $(n=327)$ cohorts. The baseline characteristics of all patients are shown in Table 1 . The training cohort consisted of 102 men and 205 women. Of these patients, $193(62.87 \%)$ were histologically diagnosed with adenocarcinoma and $187(60.91 \%)$ were treated 
Table 1: Clinical characteristics for training and validation cohort patients

\begin{tabular}{|c|c|c|c|}
\hline Baseline characteristics & $\begin{array}{l}\text { Training cohort } \\
\qquad N=\mathbf{3 0 7}\end{array}$ & $\begin{array}{l}\text { Validation cohort } \\
\qquad N=327\end{array}$ & $P$ for value \\
\hline Age (years) & $64(57-72)$ & $65(57-74)$ & 0.546 \\
\hline Sex (male) & $102(33.22)$ & $125(38.23)$ & 0.179 \\
\hline Smoking & $58(18.89)$ & $74(22.63)$ & 0.169 \\
\hline \multicolumn{4}{|l|}{ ECOG PS } \\
\hline $0-1$ & $247(80.46)$ & $266(81.35)$ & 0.791 \\
\hline $2-3$ & $60(19.54)$ & $61(18.65)$ & \\
\hline \multicolumn{4}{|l|}{ Histological type } \\
\hline Adenocarcinoma & $193(62.87)$ & $213(65.14)$ & 0.516 \\
\hline Non-adenocarcinoma & $114(37.13)$ & $114(34.86)$ & \\
\hline TNM stage (I/II/III) & $99 / 106 / 102$ & $93 / 99 / 135$ & 0.111 \\
\hline pT stage $(1 / 2 / 3 / 4)$ & $60 / 44 / 181 / 22$ & $72 / 59 / 160 / 36$ & 0.062 \\
\hline pN stage $(0 / 1 / 2 / 3)$ & $128 / 93 / 54 / 32$ & $131 / 113 / 57 / 26$ & 0.562 \\
\hline chemotherapy & $187(60.91)$ & $175(53.52)$ & 0.067 \\
\hline \multicolumn{4}{|l|}{ CEA (ng/mL) } \\
\hline$<5$ & $200(65.15)$ & $194(59.33)$ & 0.146 \\
\hline$\geq 5$ & $107(34.85)$ & $133(40.67)$ & \\
\hline TBIL $(\mu \mathrm{mol} / \mathrm{L})$ & $9.7(6.8-14.4)$ & $10.0(6.8-15.2)$ & 0.114 \\
\hline DBIL $(\mu \mathrm{mol} / \mathrm{L})$ & $3.1(2.1-4.5)$ & $3.4(2.4-5.2)$ & 0.356 \\
\hline IBIL $(\mu \mathrm{mol} / \mathrm{L})$ & $6.6(4.0-9.1)$ & $6.4(4.2-9.8)$ & 0.442 \\
\hline
\end{tabular}

ECOG PS, Eastern Cooperative Oncology Group performance status; TBIL, total bilirubin; DBIL, direct bilirubin; IBIL, indirect bilirubin.

All data were analyzed using $\chi^{2}$ test or Mann-Whitney $U$ test.

Values are expressed as medians (interquartile range) or frequencies and percentages.

with first-line chemotherapy. The mean patient age was $65 \pm 12$ years. A total of $146(47.56 \%)$ patients in the training cohort died during follow-up. The TNM stage distribution varied widely in the validation cohort: 93 patients (28.44\%) had early-stage NSCLC and 131 $(40.06 \%)$ had node-negative disease. A total of 143 $(43.73 \%)$ patients in the validation cohort died during follow-up (median follow-up period, 1,085 days).

\section{Correlation between serum bilirubin levels and the baseline characteristics of NSCLC patients}

The baseline characteristics of the patients in the training and validation cohorts were similar $(P>0.05)$ (Table 1). The X-tile software was used to identify the optimal serum bilirubin cutoff value for sorting patients in the training cohort into low and high bilirubin groups: $15 \mu \mathrm{mol} / \mathrm{L}$ for total bilirubin (TBIL), $4.5 \mu \mathrm{mol} / \mathrm{L}$ for direct bilirubin (DBIL), and $9.1 \mu \mathrm{mol} / \mathrm{L}$ for indirect bilirubin (IBIL) (Figure 1). Pretreatment DBIL levels were associated with TNM stage, $\mathrm{pT}$ stage, $\mathrm{pN}$ stage, and chemotherapy in both the training and validation cohorts (Table 3). However, no correlation between pretreatment
TBIL and IBIL levels and patient baseline characteristics was observed in either cohort (Tables 2 and 4).

\section{Association between DBIL levels and response to first-line platinum-based combination chemotherapy}

We next investigated whether pretreatment DBIL levels were associated with the response to first-line platinum-based combination chemotherapy (Table 5). Complete response was obtained after two cycles of chemotherapy in $11(7.43 \%)$ patients in the low DBIL group compared to three $(11.11 \%)$ in the high DBIL group. Partial response was also more common in the high DBIL group (51.85\% vs. 29.05\%). There were 94 patients $(63.51 \%)$ in the low DBIL group who were non-responders compared to $10(37.03 \%)$ in the high DBIL group $(P=0.010)$.

\section{Prognostic significance of serum bilirubin levels}

Univariate log-rank tests for clinical prognostic factors in the training and validation cohorts are shown 
in Table 6. The prognostic factors that were significant on univariate analysis were TNM stage, $\mathrm{pT}$ stage, $\mathrm{pN}$ stage, chemotherapy, TBIL, DBIL, and IBIL. Kaplan-Meier survival curves stratified by serum bilirubin level are shown in Figures 1 and 2 (all $P$-values $>0.05$ ). Multivariate Cox regression analysis confirmed that TNM stage, $\mathrm{pT}$ stage, $\mathrm{pN}$ stage, and DBIL were independent prognostic indicators for OS in both the training and validation cohorts.

\section{A serum bilirubin-based nomogram predicts NSCLC patient survival}

We developed a serum bilirubin-based nomogram to predict 5-year OS rates in NSCLC patients. We included pT stage, $\mathrm{pN}$ stage, chemotherapy, TBIL, DBIL, and IBIL in the nomogram for patients in both the training (Figure 3A) and validation (Figure 3B) cohorts. We found that $\mathrm{pT}$ stage and $\mathrm{pN}$ stage were poor prognostic factors for survival, whereas high DBIL was a favorable prognostic factor. There results were validated by multivariate Cox regression analysis (Table 6). Calibration curves demonstrated that the predicted and actual 5-year OS rates were similar (Figure 3C and 3D).

We next compared the predictive accuracy of our prognostic model with that of the traditional TNM staging system. The Harrell's c-indices for the nomogram were 0.714 and 0.735 compared to 0.684 and 0.701 for the TNM staging system in the training and validation cohorts, respectively. These data indicated the predictive accuracy of the DBIL-based nomogram was superior to that of the TNM staging system $(P<0.05)$.
A

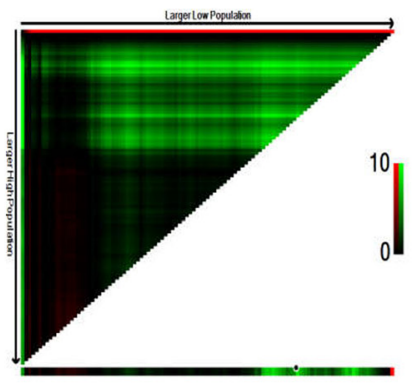

B

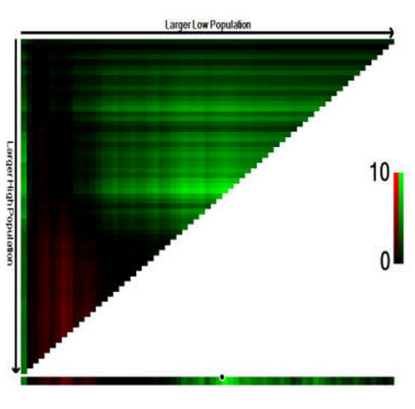

C

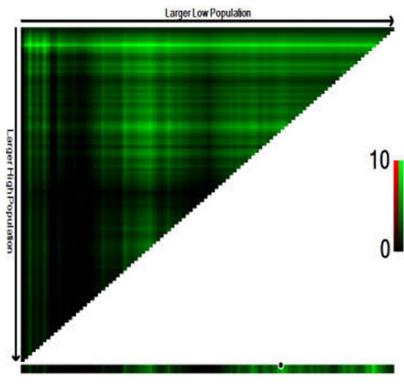

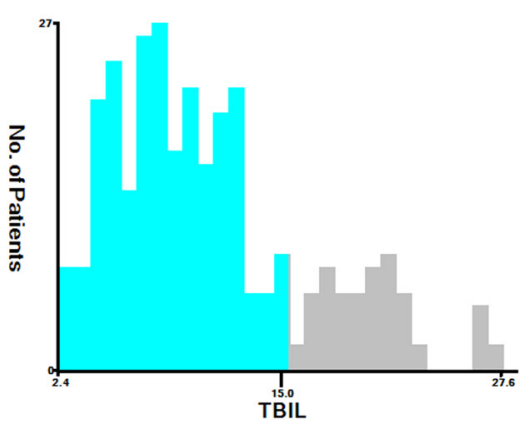
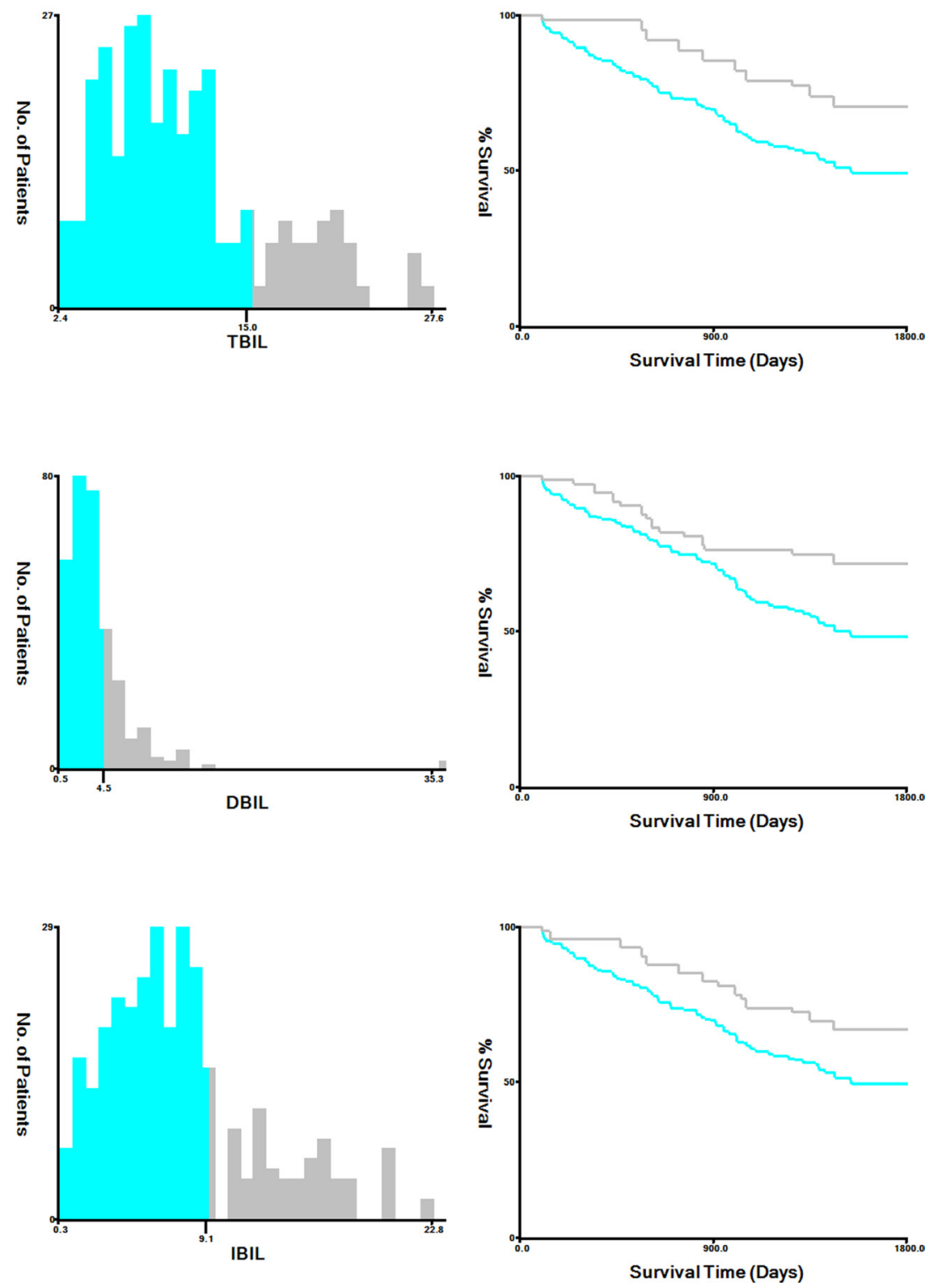

Figure 1: Analysis of TBIL (A), DBIL (B), and IBIL (C) levels in the training cohort using X-tile. X-tile plots (left panels); optimal cutoffs (black circles, middle panels); Kaplan-Meier plots (right panels). 
Table 2: Distribution of baseline characteristics stratified by pretreatment TBIL

\begin{tabular}{lcccccc}
\hline \multirow{2}{*}{ Baseline characteristics } & \multicolumn{3}{c}{ Training cohort } & \multicolumn{3}{c}{ Validation cohort } \\
\cline { 2 - 7 } & Low TBIL & High TBIL & $\boldsymbol{P}$ & Low TBIL & High TBIL & $\boldsymbol{P}$ \\
\hline Age (years) & $63(56-72)$ & $64(55-73)$ & 0.324 & $65(57-75)$ & $64(56-74)$ & 0.096 \\
Sex (male) & $81(34.47)$ & $21(29.17)$ & 0.403 & $85(35.12)$ & $40(47.06)$ & 0.051 \\
Smoking & $42(17.87)$ & $16(22.22)$ & 0.409 & $52(21.49)$ & $22(25.88)$ & 0.405 \\
ECOG PS & & & & & & \\
$\quad 0-1$ & $185(78.72)$ & $62(86.11)$ & 0.167 & $201(83.06)$ & $65(76.47)$ & 0.180 \\
$\quad$ 2-3 & $50(21.28)$ & $10(13.89)$ & & $41(16.94)$ & $20(23.53)$ & \\
Histological type & & & & & & \\
$\quad$ Adenocarcinoma & $152(64.68)$ & $41(56.94)$ & 0.235 & $162(66.94)$ & $51(60.00)$ & 0.248 \\
$\quad$ Non-adenocarcinoma & $83(35.32)$ & $31(43.06)$ & & $80(33.06)$ & $34(40.00)$ & \\
TNM stage (I/II/III) & $80 / 79 / 76$ & $19 / 27 / 26$ & 0.478 & $66 / 73 / 103$ & $27 / 26 / 32$ & 0.692 \\
pT stage (1/2/3/4) & $43 / 34 / 142 / 16$ & $17 / 10 / 39 / 6$ & 0.716 & $58 / 41 / 119 / 24$ & $14 / 18 / 41 / 12$ & 0.355 \\
pN stage (0/1/2/3) & $96 / 72 / 45 / 22$ & $32 / 21 / 9 / 10$ & 0.444 & $95 / 89 / 41 / 17$ & $36 / 24 / 16 / 9$ & 0.456 \\
chemotherapy & $149(63.40)$ & $38(52.78)$ & 0.106 & $123(50.83)$ & $52(61.18)$ & 0.100 \\
CEA (ng/mL) & & & & & & \\
$\quad<5$ & $158(67.23)$ & $42(58.33)$ & 0.166 & $138(57.02)$ & $56(65.88)$ & 0.153 \\
$\geq 5$ & $77(32.77)$ & $30(41.67)$ & & $104(42.98)$ & $29(34.12)$ & \\
\hline
\end{tabular}

ECOG PS, Eastern Cooperative Oncology Group performance status; TBIL, total bilirubin.

All data were analyzed using $\chi^{2}$ test or Mann-Whitney $U$ test.

Values are expressed as medians (interquartile range) or frequencies and percentages.

Table 3: Distribution of baseline characteristics stratified by pretreatment DBIL

\begin{tabular}{lcccccc}
\hline \multirow{2}{*}{ Baseline characteristics } & \multicolumn{3}{c}{ Training cohort } & \multicolumn{3}{c}{ Validation cohort } \\
\cline { 2 - 7 } & Low DBIL & High DBIL & $\boldsymbol{P}$ & Low DBIL & High DBIL & $\boldsymbol{P}$ \\
\hline Age (years) & $64(56-73)$ & $64(55-73)$ & 0.196 & $64(56-75)$ & $65(56-74)$ & 0.259 \\
Sex (male) & $75(33.33)$ & $27(32.93)$ & 0.947 & $68(31.34)$ & $34(30.91)$ & 0.937 \\
Smoking & $38(16.89)$ & $20(24.39)$ & 0.137 & $45(20.74)$ & $29(26.36)$ & 0.251 \\
ECOG PS & & & & & \\
$\quad 0-1$ & $180(80.00)$ & $67(81.71)$ & 0.739 & $175(80.65)$ & $91(82.73)$ & 0.648 \\
$\quad 2-3$ & $45(20.00)$ & $15(18.29)$ & & $42(19.35)$ & $19(17.27)$ & \\
Histological type & & & & & \\
$\quad$ Adenocarcinoma & $142(63.11)$ & $51(62.20)$ & 0.883 & $140(64.52)$ & $73(66.36)$ & 0.740 \\
$\quad$ Non-adenocarcinoma & $83(36.89)$ & $31(37.80)$ & & $77(35.48)$ & $37(33.64)$ & \\
TNM stage (I/II/III) & $61 / 82 / 82$ & $38 / 24 / 20$ & 0.005 & $54 / 76 / 97$ & $39 / 23 / 38$ & 0.014 \\
pT stage (1/2/3/4) & $32 / 28 / 171 / 14$ & $28 / 16 / 10 / 8$ & $<0.001$ & $42 / 25 / 128 / 22$ & $30 / 34 / 32 / 14$ & $<0.001$ \\
pN stage (0/1/2/3) & $80 / 73 / 42 / 15$ & $48 / 20 / 12 / 17$ & 0.001 & $78 / 92 / 28 / 19$ & $53 / 21 / 29 / 7$ & $<0.001$ \\
chemotherapy & $151(67.11)$ & $36(43.90)$ & $<0.001$ & $148(68.20)$ & $27(24.55)$ & $<0.001$ \\
CEA (ng/mL) & & & & & \\
$\quad<5$ & $140(62.22)$ & $60(73.17)$ & 0.075 & $135(62.21)$ & $59(53.64)$ & 0.136 \\
$\quad$ ㄷ & $85(37.78)$ & $22(26.83)$ & & $82(37.79)$ & $51(46.36)$ & \\
\hline
\end{tabular}

ECOG PS, Eastern Cooperative Oncology Group performance status; DBIL, direct bilirubin.

All data were analyzed using $\chi^{2}$ test or Mann-Whitney $U$ test.

Values are expressed as medians (interquartile range) or frequencies and percentages. 
Table 4: Distribution of baseline characteristics stratified by pretreatment IBIL

\begin{tabular}{|c|c|c|c|c|c|c|}
\hline \multirow{2}{*}{ Baseline characteristics } & \multicolumn{3}{|c|}{ Training cohort } & \multicolumn{3}{|c|}{ Validation cohort } \\
\hline & Low IBIL & High IBIL & $P$ & Low IBIL & High IBIL & $P$ \\
\hline Age (years) & $64(56-73)$ & $64(54-73)$ & 0.180 & $64(56-75)$ & $64(56-73)$ & 0.098 \\
\hline Sex (male) & 75 (33.19) & $27(33.33)$ & 0.981 & $95(41.48)$ & $30(30.61)$ & 0.064 \\
\hline Smoking & $39(17.26)$ & $19(23.46)$ & 0.221 & $49(21.40)$ & $25(25.51)$ & 0.415 \\
\hline \multicolumn{7}{|l|}{ ECOG PS } \\
\hline $0-1$ & $142(62.83)$ & $55(67.90)$ & 0.414 & $180(78.60)$ & $86(87.76)$ & 0.052 \\
\hline $2-3$ & $84(37.17)$ & $26(32.10)$ & & $49(21.40)$ & $12(12.24)$ & \\
\hline \multicolumn{7}{|l|}{ Histological type } \\
\hline Adenocarcinoma & $145(64.16)$ & $48(59.26)$ & 0.434 & $151(65.94)$ & $62(63.27)$ & 0.642 \\
\hline Non-adenocarcinoma & $81(35.84)$ & $33(40.74)$ & & $78(34.06)$ & $36(36.73)$ & \\
\hline TNM stage (I/II/III) & $78 / 78 / 70$ & $21 / 28 / 32$ & 0.263 & $67 / 70 / 92$ & $26 / 29 / 43$ & 0.807 \\
\hline pT stage $(1 / 2 / 3 / 4)$ & $50 / 32 / 131 / 13$ & $10 / 12 / 50 / 9$ & 0.141 & $52 / 32 / 116 / 29$ & $20 / 27 / 44 / 7$ & 0.023 \\
\hline pN stage $(0 / 1 / 2 / 3)$ & $98 / 73 / 32 / 23$ & $30 / 20 / 22 / 9$ & 0.058 & $84 / 84 / 39 / 22$ & $47 / 29 / 18 / 4$ & 0.117 \\
\hline chemotherapy & $144(63.72)$ & 43 (53.09) & 0.093 & $149(65.07)$ & $26(26.53)$ & $<0.001$ \\
\hline \multicolumn{7}{|l|}{ CEA (ng/mL) } \\
\hline$<5$ & $148(65.49)$ & $52(64.20)$ & 0.835 & $140(61.14)$ & $54(55.10)$ & 0.309 \\
\hline$\geq 5$ & $78(34.51)$ & $29(35.80)$ & & $89(38.86)$ & $44(44.90)$ & \\
\hline
\end{tabular}

ECOG PS, Eastern Cooperative Oncology Group performance status; IBIL, indirect bilirubin.

All data were analyzed using $\chi^{2}$ test or Mann-Whitney $U$ test.

Values are expressed as medians (interquartile range) or frequencies and percentages.

Table 5: Treatment response in 327 NSCLC patients treated with first-line platinum-based chemotherapy

\begin{tabular}{lccc}
\hline \multirow{2}{*}{ Treatment response } & \multicolumn{2}{c}{ DBIL } & \multirow{2}{*}{$\boldsymbol{P}$} \\
\cline { 2 - 4 } & Low DBIL $(\boldsymbol{n}=\mathbf{1 4 8})$ & High DBIL $(\boldsymbol{n}=\mathbf{2 7})$ & 0.045 \\
$\mathrm{CR}$ & $11(7.43)$ & $3(11.11)$ & $14(51.85)$ \\
$\mathrm{SD}$ & $43(29.05)$ & $4(14.81)$ & $6(22.22)$ \\
$\mathrm{PD}$ & $59(39.86)$ & $17(62.96)$ & 0.010 \\
Responder (CR and PR) & $35(23.65)$ & $10(37.03)$ & \\
Non-responder (SD and PD) & $54(36.49)$ & $94(63.51)$ & \\
\hline
\end{tabular}

CR complete response, $\mathrm{PR}$ partial response, SD stable disease, $\mathrm{PD}$ progressive disease.

All data were analyzed using $\chi^{2}$ test.

\section{DISCUSSION}

We found that DBIL levels are prognostic in NSCLC. Elevated pretreatment DBIL levels were correlated with longer $\mathrm{OS}$ in both the training and validation cohorts. Additionally, elevated DBIL levels were associated with response to first-line platinum-based combination chemotherapy in the validation cohort.

Previous studies have yielded conflicting results regarding the association between serum bilirubin levels and patient prognosis in NSCLC. We found that elevated (but within the normal range) DBIL levels were associated with favorable prognosis in NSCLC, which may be explained by the anti-inflammatory, anti-oxidative, and anti-proliferative effects of bilirubin [10]. Interestingly, Horsfall et al. demonstrated that higher serum bilirubin levels were associated with a lower risk of respiratory disease and all-cause mortality [8]. In contrast, Zhang et al. found that elevated DBIL levels were associated with poor outcomes following surgery in colorectal cancer patients [11]. Although Li et al. found that elevated DBIL levels were associated with longer OS in NSCLC, pretreatment DBIL level was not an independent predictor [12]. Finally, Zhang et al. reported that pretreatment DBIL 
Table 6: Univariate and multivariate analyses of prognostic value of serum bilirubin

\begin{tabular}{|c|c|c|c|c|c|c|}
\hline \multirow{3}{*}{ Factors } & \multicolumn{3}{|c|}{ Training cohort } & \multicolumn{3}{|c|}{ Validation cohort } \\
\hline & \multirow{2}{*}{$\begin{array}{c}\begin{array}{c}\text { Univariate } \\
\text { analysis }\end{array} \\
P\end{array}$} & \multicolumn{2}{|c|}{ Multivariate analysis } & \multirow{2}{*}{$\begin{array}{c}\begin{array}{c}\text { Univariate } \\
\text { analysis }\end{array} \\
P\end{array}$} & \multicolumn{2}{|c|}{ Multivariate analysis } \\
\hline & & HR $(95 \%$ CI $)$ & $P$ & & HR (95\%CI) & $P$ \\
\hline Age (years) & 0.085 & & & 0.234 & & \\
\hline Sex (male) & 0.124 & & & 0.162 & & \\
\hline Smoking & 0.541 & & & 0.324 & & \\
\hline ECOG PS (2-3) & 0.594 & & & 0.160 & & \\
\hline Histological type & 0.220 & & & 0.108 & & \\
\hline TNM stage & $<0.001$ & & & $<0.001$ & & \\
\hline I & & Reference & & & Reference & \\
\hline II & & $1.24(0.96-3.25)$ & 0.057 & & $1.30(1.11-2.64)$ & 0.020 \\
\hline III & & $2.59(1.20-4.98)$ & $<0.001$ & & $2.42(1.14-3.58)$ & $<0.001$ \\
\hline pT stage & $<0.001$ & & & $<0.001$ & & \\
\hline $\mathrm{T} 1-2$ & & Reference & & & Reference & \\
\hline T3-4 & & $2.45(1.22-5.19)$ & $<0.001$ & & $1.99(1.12-3.20)$ & 0.002 \\
\hline $\mathrm{pN}$ stage & $<0.001$ & & & $<0.001$ & & \\
\hline $\mathrm{T} 0-1$ & & Reference & & & Reference & \\
\hline $\mathrm{T} 2-3$ & & $1.86(1.08-2.80)$ & 0.005 & & $2.00(1.20-3.52)$ & $<0.001$ \\
\hline chemotherapy & 0.042 & $1.14(0.95-1.86)$ & 0.089 & 0.030 & $1.23(0.82-1.64)$ & 0.124 \\
\hline CEA (abnormal) & 0.385 & & & 0.110 & & \\
\hline TBIL $(\geq 15 \mu \mathrm{mol} / \mathrm{L})$ & 0.005 & $0.98(0.76-1.24)$ & 0.582 & 0.012 & $1.01(0.65-1.09)$ & 0.101 \\
\hline DBIL $(\geq 4.5 \mu \mathrm{mol} / \mathrm{L})$ & $<0.001$ & $0.64(0.52-0.91)$ & 0.004 & 0.004 & $0.74(0.61-0.88)$ & $<0.001$ \\
\hline IBIL $(\geq 9.1 \mu \mathrm{mol} / \mathrm{L})$ & 0.032 & $1.00(0.80-1.35)$ & 0.532 & 0.001 & $0.94(0.56-1.02)$ & 0.098 \\
\hline
\end{tabular}

ECOG PS, Eastern Cooperative Oncology Group performance status; TBIL, total bilirubin; DBIL, direct bilirubin; IBIL, indirect bilirubin.

was positively correlated with OS in NSCLC patients with EGFR mutations [13].

Our results indicated that reduced DBIL levels were correlated with advanced NSCLC and poor prognosis. Serum DBIL was found to be an independent prognostic indicator in both our retrospective training and prospective validation cohorts. Collectively, the data suggest that serum DBIL levels are prognostic in NSCLC.

Although TNM stage is a useful prognostic indicator, it may not be adequate for predicting survival. The predictive power could be improved by combining TNM stage with other biomarkers into a single index [14-16]. Therefore, we established a nomogram based on several pathological characteristics and evaluated whether it could accurately predict prognosis in NSCLC. We found that the prognostic model accurately predicted 5-year OS in both the training and validation cohorts. Serum DBIL level was incorporated into the model through a stepwise algorithm and the predictive power verified using calibration curves. Importantly, the nomogram was superior to the TNM staging system in predicting patient prognosis.
Our study had several strengths. First, all of the NSCLC patients were treated at a single center. Second, the analysis included both retrospective and prospective cohorts. Finally, the X-tile software was used to identify the optimal cutoff value for serum bilirubin [17]. Our study also had several limitations including the small sample size. Additionally, we only included pretreatment serum bilirubin levels in the analysis and did not test whether alterations in serum bilirubin levels throughout treatment could impact patient survival.

In summary, we have demonstrated that serum DBIL levels are prognostic in NSCLC. A DBIL-based nomogram is more accurate than the TNM staging system in predicting clinical outcomes in NSCLC.

\section{MATERIALS AND METHODS}

\section{Study patients}

Consecutive patients who were treated in the Department of Respiratory Medicine and Gerontology at Huai'an First People's Hospital between January 2006 and 
A

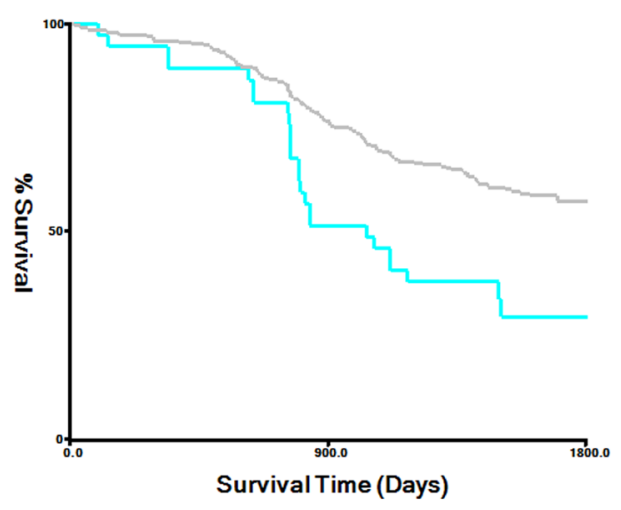

B

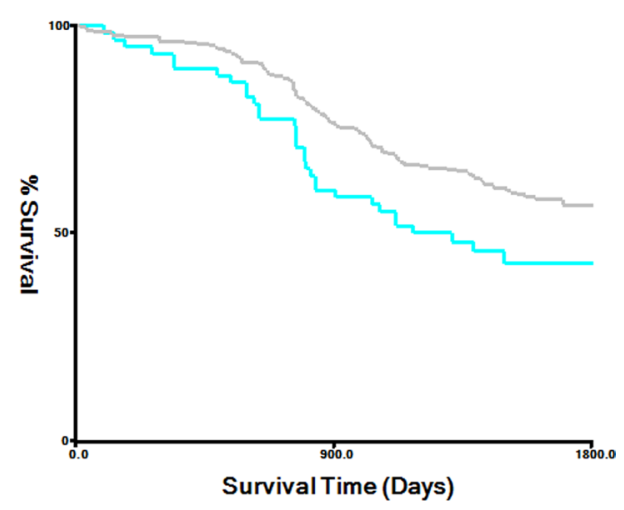

C

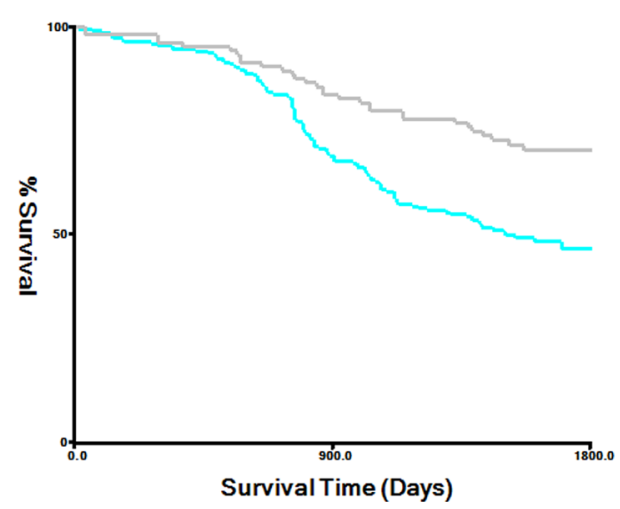

Figure 2: Kaplan-Meier survival curves stratified by serum bilirubin level in the validation cohort.

A

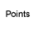

"

DBIL

Tota Panits

3-year sunviva

5-year sunval

$\mathrm{C}$

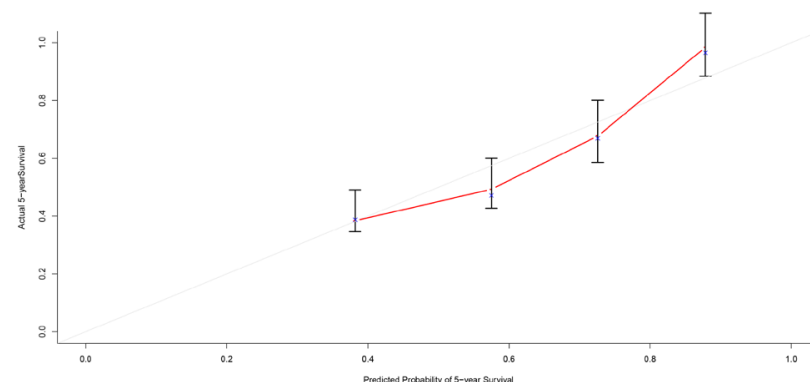

B

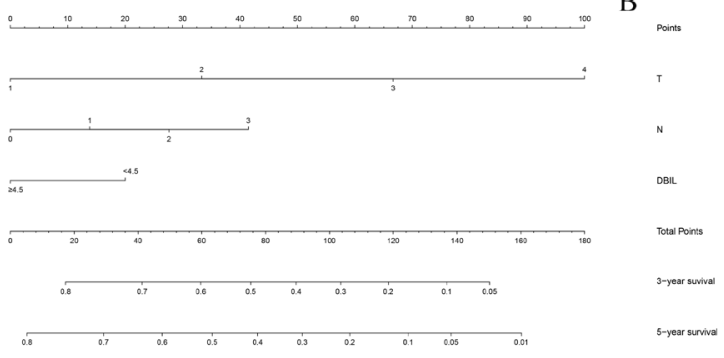

D

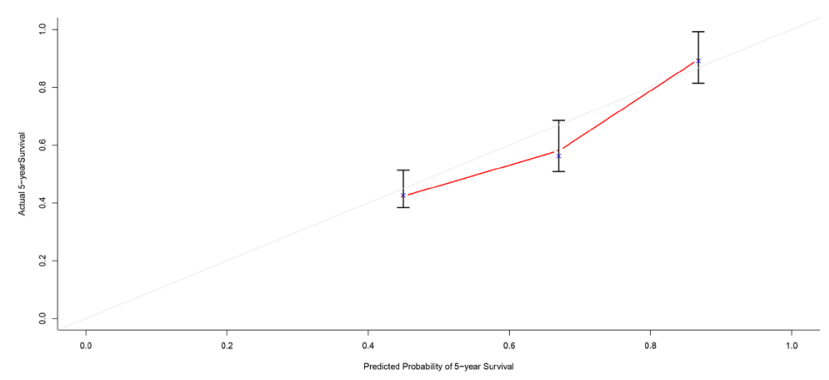

Figure 3: Prognostic nomogram for predicting survival in NSCLC patients. The c-index values were 0.714 for the training cohort (A) and 0.735 for the validation cohort (B). Calibration curves for 3-year and 5-year overall survival for the training cohort (C) and the validation cohort $(\mathbf{D})$. 
December 2013 were enrolled in the study. The retrospective training cohort included 307 patients who underwent surgical resection between January 2006 and December 2009. The prospective validation cohort consisted of 327 patients who underwent surgical resection between January 2010 and December 2013. The diagnosis of NSCLC was confirmed histologically according to the TNM criteria (2009 AJCC criteria). All protocols were approved by the Ethics Committee of Nanjing Medical University.

Eligible patients had (1) TNM stage: I-IIIA NSCLC, (2) were not treated prior to serum collection, and (3) had complete follow-up information available. Patients were excluded if they had a history of other malignancies, hepatobiliary or hemolytic disease, and/or insufficient follow-up information available. Lab tests were performed before treatment to establish baseline bilirubin levels.

\section{Data collection and measurement of serum bilirubin levels}

Baseline characteristics including age, sex, and smoking history were obtained through a structured questionnaire. Pathological, treatment response, and follow-up data were evaluated by physicians. Serum bilirubin levels (TBIL, DBIL, and IBIL) were measured in fasting blood samples prior to treatment using the vanadium oxidation method.

\section{Statistical analysis}

Continuous data are presented as the mean \pm standard deviation (SD) or median and interquartile range (IQR). Categorical data are expressed as percentages. The $\mathrm{X}$-tile software was used to identify the optimal serum bilirubin cutoff level for sorting patients based on bilirubin levels. Differences between two groups were analyzed using $\chi^{2}$ or Mann-Whitney $U$ tests. Survival analysis was performed using Kaplan-Meier curves and log-rank tests. Multivariate analysis was performed using a Cox proportional hazards model. The serum bilirubin-based nomogram was established using a stepwise algorithm and the R software. The prognostic accuracy of the nomogram was compared to that of the TNM staging system using Harrell's c-index. $P$ values $<0.05$ were considered statistically significant.

\section{Author contributions}

Conceived of and designed the experiments: LXW and YJS; Performed the experiments: YJS, XHG and YQH; Analyzed the data: YJS and XHG; Contributed reagents/materials/analysis tools: LXW and $\mathrm{YQH}$; Wrote the manuscript: YJS.

\section{ACKNOWLEDGMENTS}

None.

\section{CONFLICTS OF INTEREST}

The authors declare that there are no conflicts of interest.

\section{REFERENCES}

1. Siegel RL, Miller KD, Jemal A. Cancer statistics, 2016. CA Cancer J Clin. 2016; 66:7-30. https://doi.org/10.3322/ caac. 21332.

2. Herbst RS, Heymach JV, Lippman SM. Lung cancer. N Engl J Med. 2008; 359:1367-80. https://doi.org/10.1056/ NEJMra0802714.

3. Hartgrink HH, Jansen EP, van Grieken NC, van de Velde CJ. Gastric cancer. Lancet. 2009; 374:477-90. https:// doi.org/10.1016/S0140-6736(09)60617-6.

4. Tanner NT, Sherman CA, Silvestri GA. Biomarkers in the selection of maintenance therapy in non-small cell lung cancer. Transl Lung Cancer Res. 2012; 1:96-8. https://doi. org/10.3978/j.issn.2218-6751.2012.03.02.

5. Wei J, Zhao H, Fan G, Li J. Bilirubin treatment suppresses pulmonary inflammation in a rat model of smoke-induced emphysema. Biochem Biophys Res Commun. 2015; 465:180-7. https://doi.org/10.1016/j.bbrc.2015.07.133.

6. O’Malley SS, Wu R, Mayne ST, Jatlow PI. Smoking cessation is followed by increases in serum bilirubin, an endogenous antioxidant associated with lower risk of lung cancer and cardiovascular disease. Nicotine Tob Res. 2014; 16:1145-9. https://doi.org/10.1093/ntr/ntu067.

7. Temme EH, Zhang J, Schouten EG, Kesteloot H. Serum bilirubin and 10-year mortality risk in a Belgian population. Cancer Causes Control. 2001; 12:887-94.

8. Horsfall LJ, Rait G, Walters K, Swallow DM, Pereira SP, Nazareth I, Petersen I. Serum bilirubin and risk of respiratory disease and death. JAMA. 2011; 305:691-7. https://doi.org/10.1001/jama.2011.124.

9. Wen CP, Zhang F, Liang D, Wen C, Gu J, Skinner H, Chow WH, Ye Y, Pu X, Hildebrandt MA, Huang M, Chen $\mathrm{CH}$, Hsiung $\mathrm{CA}$, et al. The ability of bilirubin in identifying smokers with higher risk of lung cancer: a large cohort study in conjunction with global metabolomic profiling. Clin Cancer Res. 2015; 21:193-200. https://doi. org/10.1158/1078-0432.CCR-14-0748.

10. Marnett LJ. Oxyradicals and DNA damage. Carcinogenesis. 2000; 21:361-70.

11. Zhang Q, Ma X, Xu Q, Qin J, Wang Y, Liu Q, Wang H, Li M. Nomograms incorporated serum direct bilirubin level for predicting prognosis in stages II and III colorectal cancer after radical resection. Oncotarget. 2017; 8:71138-46. https://doi.org/10.18632/oncotarget.11424.

12. Li N, Xu M, Cai MY, Zhou F, Li CF, Wang BX, Ou W, Wang SY. Elevated serum bilirubin levels are associated with improved survival in patients with curatively resected non-small-cell lung cancer. Cancer Epidemiol. 2015; 39:763-8. https://doi.org/10.1016/j.canep.2015.06.007. 
13. Zhang Y, Xu J, Lou Y, Hu S, Yu K, Li R, Zhang X, Jin B, Han B. Pretreatment direct bilirubin and total cholesterol are significant predictors of overall survival in advanced non-small-cell lung cancer patients with EGFR mutations. Int J Cancer. 2017; 140:1645-52. https://doi.org/10.1002/ ijc.30581.

14. Boonpipattanapong T, Chewatanakornkul S. Preoperative carcinoembryonic antigen and albumin in predicting survival in patients with colon and rectal carcinomas. J Clin Gastroenterol. 2006; 40:592-5.

15. Deng QW, Wang H, Sun CZ, Xing FL, Zhang HQ, Zuo L, $\mathrm{Gu}$ ZT, Yan FL. Triglyceride to high-density lipoprotein cholesterol ratio predicts worse outcomes after acute ischaemic stroke. Eur J Neurol. 2017; 24:283-91. https:// doi.org/10.1111/ene.13198.

16. Deng QW, Li S, Wang H, Zuo L, Zhang HQ, Gu ZT, Xing FL, Yan FL. The short-term prognostic value of the triglyceride-tohigh-density lipoprotein cholesterol ratio in acute ischemic stroke. Aging Dis. 2017 Jul 10. http://www. aginganddisease.org/EN/10.14336/AD.2017.0629. [Epub ahead of print].

17. Camp RL, Dolled-Filhart M, Rimm DL. X-tile: a new bioinformatics tool for biomarker assessment and outcomebased cut-point optimization. Clin Cancer Res. 2004; 10:7252-9. https://doi.org/10.1158/1078-0432.CCR-04-0713. 